\title{
DEVELOPMENT OF SYNERGETIC-ACTIVE SENSOR-SYSTEM FOR EVALUATION OF OBSERVATIONS BY EARTHCARE
}

\author{
Hajime Okamoto $^{{ }^{*}}$, Kaori Sato ${ }^{1}$, Masahiro Fujikawa ${ }^{1}$, Eiji Oikawa ${ }^{1}$, Tomoaki Nishizawa ${ }^{2}$, Shoken \\ Ishii $^{3}$, Yoshitaka Jin ${ }^{2}$, Makoto Aoki ${ }^{3}$, Nobuo Sugimoto ${ }^{2}$ \\ ${ }^{1}$ Research Institute for Applied Mechanics, Kyushu University, Kasuga Fukuoka 816-8580, Japan \\ ${ }^{2}$ National Institute for Environmental Studies, Tsukuba Ibaraki 305-8506, Japan \\ ${ }^{3}$ National Institute of Information and Communications Technology, Koganei Tokyo 184-8795, Japan
}

\begin{abstract}
We develop the synergetic ground-based activesensor-system for the evaluation of observations by space-borne lidars. The system consists of second version of multi-field-view multiplescattering polarization lidar (MFMSPL-2), multiple-field-of-view high spectral resolution polarization lidar, direct-detection Doppler wind lidar, coherent Doppler wind lidar and $94 \mathrm{GHz}$ cloud profiling radar. The system can simulate observed signals from sensors onboard the joint Japanese/European mission Earth Clouds, Aerosols and Radiation Explorer (EarthCARE). The observation system can provide unique opportunity to study interaction of cloud microphysics, aerosol microphysics, vertical air motion and vertical distribution of horizontal wind and it will lead to evaluate cloud-convective parameterization and to reduce uncertainties in climate change predictions.
\end{abstract}

\section{INTRODUCTION}

Recently, we developed the first version of the multi-field-view multiple-scattering polarization lidar system (MFMSPL) by combining a $10 \mathrm{mrad}-$ FOV and a polarization function with a total FOV $>35$ mrad [1]. The MFMSPL-1 has eight channels (four parallel and four perpendicular). The FOV of each telescope was chosen to be $10 \mathrm{mrad}$. A wavelength $532 \mathrm{~nm}$ was detected. There were four tilt angles, $0,10,20$, and $30 \mathrm{mrad}$ used to detect signals. The lidar was designed to detect lidar signals from optically thicker parts of clouds that a conventional lidar could not penetrate. It is also designed to detect similar magnitude of lidar signals influenced by multiple scattering detected by space-borne lidars such as Cloud-Aerosol Lidar with orthogonal Polarization
(CALIPSO). Calibration procedure was described in [1]. Depolarization ratio values of low-level optically thick water clouds detected by the MFMSPL were as large as those detected by CALIPSO.

Recent theoretical achievement to treat lidar multiple scatterings was as follows. A practical model (called Physical Model: PM) for accounting lidar attenuated backscattering coefficient (ß) was developed for global spaceborne lidar data [2]. Higher-order phase function was analytically derived to drastically reduce computational cost for simulating the angular distribution of the multiple scattering irradiance. The decay rate of the multiple scattering backscattered irradiance was given based on the path integral approach. The estimated $\beta$ by the PM was in good agreement with Monte Carlo simulations. The PM was extended with a polarization function to create a vectorized physical model (VPM) to analyze vertical profile of the observed depolarization ratio due to multiple scattering from water clouds by spaceborne lidar [3]. The VPM incorporates a highorder scattering matrix and accommodates mechanics that modify the polarization state during multiple scattering events. The estimated depolarization ratio by the VPM showed good agreement with vectorized Monte Carlo simulations. VPM can simulate polarization properties under multiple scattering conditions effectively for the first time.

Japanese/European joint mission EarthCARE is scheduled to be launched in 2021. It will provide global profiles of microphysics of clouds, aerosols, rain and snow with their radiative fluxes and heating rates [4]. It will carry four instruments; (1) Atmospheric lidar (ATLID), (2) Cloud profiling radar (CPR), (3) Multispectral imager (MSI) and 
(4) Broad band radiometer (BBR). ATLID is a $355-\mathrm{nm}$ high spectral resolution lidar so that extinction coefficient and backscattering coefficient will be directly derived. Depolarization ratio will be also observed. Its foot print size is about $30 \mathrm{~m}$ that is three times smaller than that of CALIPSO. Thus less degree of multiple scattering for ATLID is expected compared with CALIPSO for the same clouds. A two-dimensional diagram of lidar ratio and depolarization ratio was proved to be effective for discriminating among ice particle types and orientations [5].

In a frame of the MFMSPL-concept, we develop the 355-nm-MFMSPL-2 and HSRL in order to quantify the EarthCARE and Direct detection Doppler lidar at $355 \mathrm{~nm}$. Evaluation of EarthCARE and Aeolus algorithms is possible by the synergetic active sensor systems.

\section{Synergetic active sensor system}

MFMSPL-2 has ten channels, five parallel and five perpendicular. Five telescopes are used instead of ten telescopes by using polarization cube. The wavelength of $355 \mathrm{~nm}$ is detected.

High spectral resolution lidar is developed. It has narrow (1mrad) and wide (10mrad) FOV to study multiple scattering effect in the backscattering and extinction retrievals in cloud observations. Wavelengths of $355 \mathrm{~nm}$ and $532 \mathrm{~nm}$ are panned to be detected. 532nm-HSRL system was completed. 355-nm-HSRL system and 355-nm-directdetection Doppler lidar are under development.

\section{RESULTS}

Observation of low and high clouds by MFMSPL2 was conducted on 23 October 2018 in Koganei, Tokyo, Japan. Attenuated backscattering coefficient detected by vertical point channel (CH.1) showed that the values of low-level clouds located at $5 \mathrm{~km}$ at $17,18.5$ and 20 UTC exceeded $10^{-4}[1 / \mathrm{m} / \mathrm{sr}]$ (Fig. 1a).

Attenuated backscattering coefficient of upper clouds located between 8 to $10 \mathrm{~km}$, were around $10-5[1 / \mathrm{m} / \mathrm{sr}]$. Depolarization ratio of low-level clouds was 0.2-0.3 at vertical channel (Fig.1b). Upper clouds show depolarization ratio $>0.4$, indicating ice clouds. Same clouds detected by off-beam channel (CH3), whose tilt angle is 10 mrad, showed smaller backscattering than for vertical channel (Fig,2a). Attenuated backscattering coefficient of low-level water clouds detected by off-beam channel showed larger values than those of ice clouds due to the large optical thickness and large contribution of multiple scattering component .

Depolarization ratio of water clouds detected by CH.3 and CH.4 ranged from 0.3 to 0.6 (Fig,2b). These values were larger than those by $\mathrm{CH} .1$ and CH.2 and the increase was attributed to multiple scattering in optically thick water clouds. Depolarization of ice clouds revealed two different features. Thick ice clouds observed between 17 and 17.5 UTC showed significantly large depolarization ratio $(>0.5)$ for off-beam channels. The value is larger than the value detected by vertical channels. Ice clouds detected after 18 UTC in off-mean channel produced small backscattering and depolarization ratio was not detected. These differences can be explained by the difference in optical thickness.

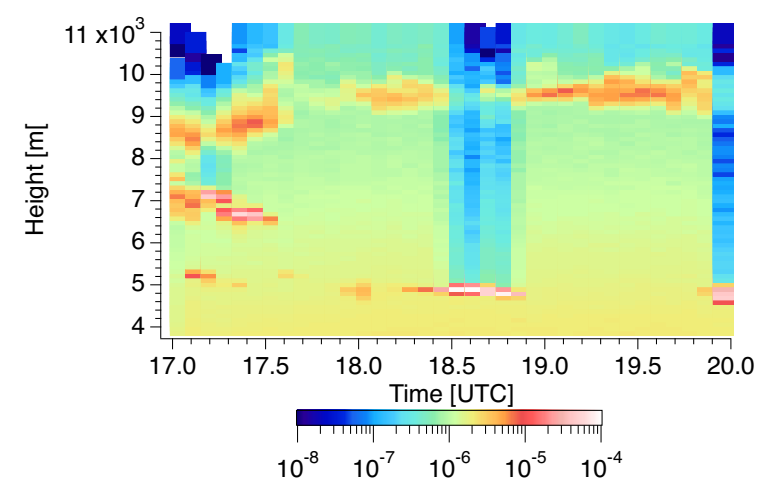

Attenuated backscattering coefficient $[1 / \mathrm{m} / \mathrm{sr}]$ at $\mathrm{CH} .1$

(a) 


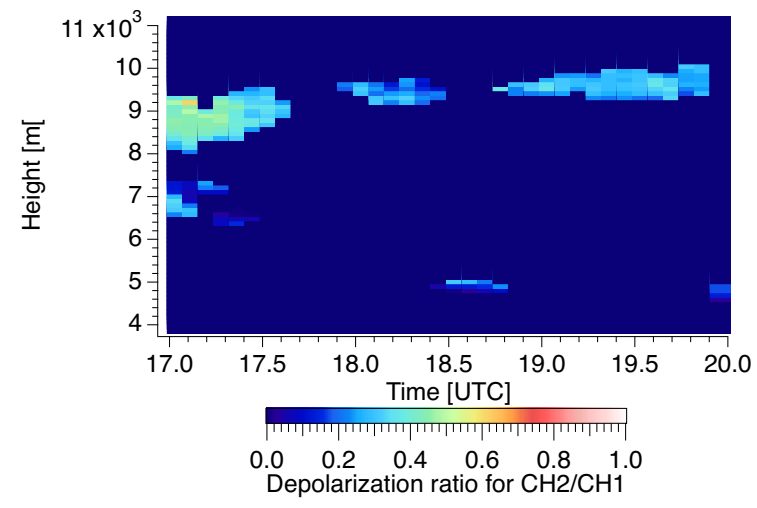

(b)

Fig. 1 (a) Time-height cross section of total attenuated backscattering coefficient of clouds and aerosols by on-beam channel, CH.1. Wavelength is $355 \mathrm{~nm}$ in 23 October 2018. (b) Same as (a) but for depolarization ratio by CH.1 and $\mathrm{CH} 2$.

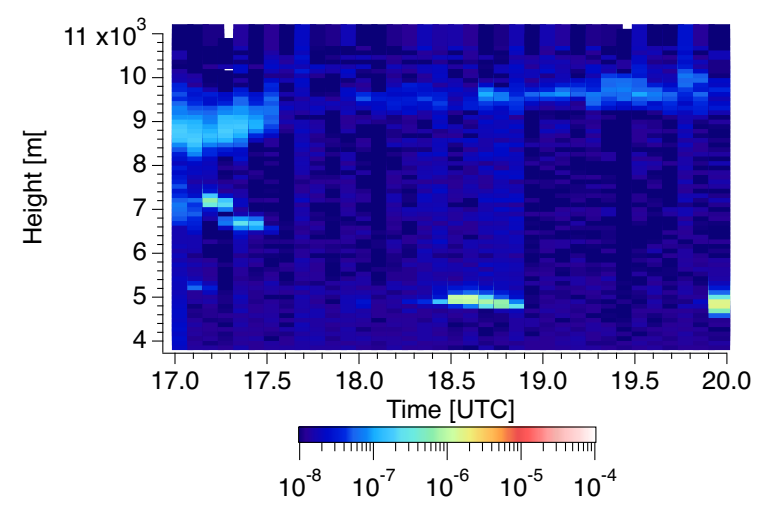

Attenuated backscattering coefficient $[1 / \mathrm{m} / \mathrm{sr}]$ at $\mathrm{CH} .3$

(a)

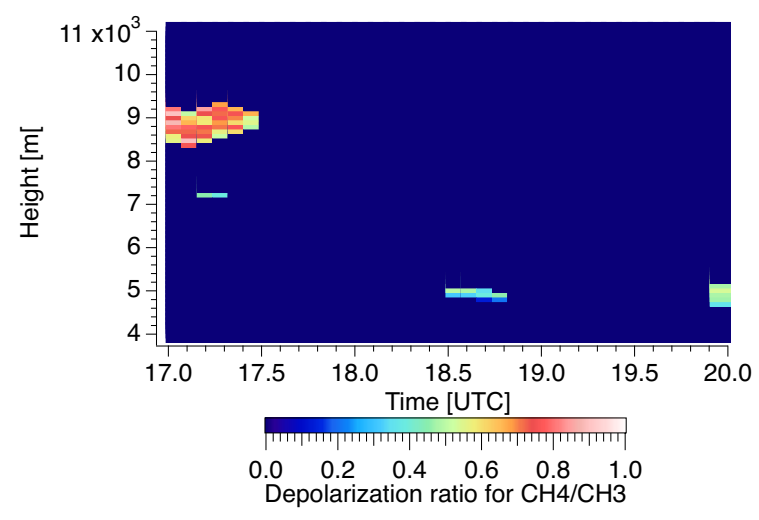

(b)
Fig.2 Same as Fig.1 but for off-beam channel. (a) attenuated backscattering coefficient for $\mathrm{CH} .3$ and (b) depolarization ratio for tilt angle of $10 \mathrm{mrad}$ by $\mathrm{CH} .3$ and CH.4.

\section{SUMMARRY}

Ice and water clouds were observed by the MFMSPL-2 at $355 \mathrm{~nm}$. Results showed increase of depolarization for both of water and ice clouds when optical thickness in off-beam channel compared with on-beam values. This indicated backscattering coefficient as well as depolarization ratio are affected by multiple scattering in space-borne lidar such as CALIPSO lidar, ATLID and Aeolus. It is important to take into account FOV dependent multiple scattering contribution in the observed values to derive cloud microphysics. Multiple field view-HSRL will possibly provide further information of the effect and synergetic use of these two new lidars will help to develop algorithms for EarthCARE and Aeolus satellites.

\section{ACKNOWLEDGEMENTS}

This work was supported by the JSPS KAKENHI JP17H06139 and JP18K03745; by the Collaborated Research Program of Research Institute for Applied Mechanics, Kyushu University; the Japan Aerospace Exploration Agency for EarthCARE Research Announcement; The Arctic Challenge for Sustainability (ArCS).

\section{REFERENCES}

[1] H. Okamoto, et al. Opt. Express 24(26), 3005330067 (2016)

[2] K. Sato, et al. Opt. Express 26(6), A301-A319 (2018)

[3] K. Sato, et al. Opt. Express 27(4), A117-A132 (2019)

[4] A. Illingworth, et al. BAMS 96(8), 1311-1332 (2015)

[5] H. Okamoto, et al. Opt. Express 27(25), 3658736600 (2019) 